\title{
Quasicrystalline Electronic States of a One-Dimensionally Modulated Ag Film
}

\author{
P. Moras, ${ }^{1}$ W. Theis, ${ }^{2}$ L. Ferrari, ${ }^{1, *}$ S. Gardonio, ${ }^{1}$ J. Fujii, ${ }^{3}$ K. Horn,${ }^{4}$ and C. Carbone ${ }^{1}$ \\ ${ }^{1}$ Istituto di Struttura della Materia, Consiglio Nazionale delle Ricerche, Trieste, Italy \\ ${ }^{2}$ Institut für Experimentalphysik, Freie Universität Berlin, Germany \\ ${ }^{3}$ TASC, Istituto Nazionale di Fisica della Materia, Consiglio Nazionale delle Ricerche, Trieste, Italy \\ ${ }^{4}$ Fritz-Haber Institut der Max-Planck Gesellschaft, Berlin, Germany
}

(Received 25 November 2005; published 17 April 2006)

\begin{abstract}
Ag films on GaAs(110) exhibit a one-dimensional quasiperiodic modulation, resulting in a Fibonacci sequence of parallel stripes with two different widths. Valence level photoemission shows that the Ag electronic states acquire a unique character along the quasiperiodic direction, distinctively manifested by a hierarchy of energy level replicas and avoided crossings at characteristic intervals in reciprocal space. These observations demonstrate the strong influence of the one-dimensional quasiperiodic potential on the Ag film states.
\end{abstract}

DOI: 10.1103/PhysRevLett.96.156401

The discovery of quasicrystalline ordering in solids in 1984 [1] has initiated the study of the physics of aperiodic order, such as the behavior of electrons, phonons, and phason defects in quasicrystals [2]. In this context, a long-standing question in solid state physics is how the conventional band picture, accounting for the electronic structure and other quasiparticle properties, can be adapted to quasiperiodic (e.g., modulated) systems. Bloch's theorem describing the electronic bands in solid matter is based upon the discrete translational periodicity of the crystal lattice. In a periodic potential, bands are formed with a continuous set of wave vectors $\vec{k}$ and eigenvalues $E(\vec{k})$, with $E(\vec{k})=E(\vec{k}+\vec{G})$ for each Bragg vector $\vec{G}$. In quasiperiodic systems, an infinite set of reciprocal lattice vectors exists such that Bragg peaks densely fill reciprocal space, and electron wave vectors and bands lose their conventional significance. The same is true for low-symmetry and low-dimensional systems with charge or spin density waves which are defined by incommensurate coexisting periodicities and lack translational invariance. Again, Bragg peaks given by all possible linear combinations of the basis $\vec{G}$ vectors then densely fill the reciprocal space along at least one direction.

The effect of aperiodic order on the electronic states has recently been explored by photoemission on quasicrystals $[3,4]$, monolayers $[5,6]$, and one-dimensional compounds [7]. Angular-resolved photoemission is uniquely capable of probing energy-momentum relations and thus of providing insight into the character of the wave functions. In spite of the absence of full translational periodicity in quasicrystals, predominant Fourier components appear to be associated with specific states, resulting in a dispersive behavior. Angle-resolved photoemission demonstrates that, in decagonal quasicrystals, electronic states exist which exhibit a delocalized character and a band dispersion with the symmetry of the quasiperiodic lattice $[3,4]$. A similar situation can be encountered in low-dimensional systems modulated by incommensurate periods. A Ag monolayer with an incommensurate Moiré superstructure on $\mathrm{Cu}(111)$ displays
PACS numbers: 71.23.Ft, 61.44.Br, 73.21.Fg, 79.60.Dp

a well defined surface state, opening a gap at the Brillouin zone boundaries [5]. A smearing of dispersion relations was observed for an incommensurate $\mathrm{Pb}$ monolayer on $\operatorname{Ag}(111)$ [6], possibly due to the spectral weight of dense and repeatedly folded bands. Information on the relative strengths of competing potentials was also derived from the spectral weights and sizes of the hybridization gap in the one-dimensional compound $\left(\mathrm{TaSe}_{4}\right)_{2} \mathrm{I}$ [7]. Model calculations based on nearly free one-dimensional electrons, and experimental findings consistently identify dispersing band states in this system. On the basis of these results, a qualitatively similar electronic structure with dispersing states and signatures of incommensurate periodicities is also predicted for a one-dimensional quasicrystal [7], but this has so far not been observed.

In order to shed light on the influence of quasicrystalline order on the electronic states in a low-dimensional system, we have studied, by angle-resolved photoemission, the electronic structure in a single-element metallic film with a simple $s-p$ band structure. Ag films on GaAs(110) present an unusual structural arrangement approaching the realization of a one-dimensional quasicrystal. A study of this system is likely to facilitate a theoretical treatment of quasicrystal formation; generally, quasicrystalline materials are three component alloys with a complex local structure [8]. We find the quasicrystalline modulation gives rise to a complex band structure, which displays intense quasiperiodic replicas in reciprocal space, in contrast to the repeated zone scheme of the electronic bands in crystalline materials.

The experiment was carried out on the VUV and APE beamlines at the Elettra synchrotron in Trieste. The substrate was obtained by in situ cleaving a GaAs(110) crystal. $\mathrm{Ag}$ films of thickness between 19 and $35 \AA$ were evaporated on the GaAs substrate held at $140 \mathrm{~K}$. Post-deposition annealing to room temperature produces atomically flat $\mathrm{Ag}$ films, if the deposition exceeds a critical amount equivalent to about $15 \AA$ average thickness [9]. The structure of $\mathrm{Ag}$ films on $\operatorname{GaAs}(110)$ has been extensively characterized in 
previous studies by means of various surface sensitive techniques. STM images on these Ag films show that the films have a remarkably uniform atomic thickness $[9,10]$. Although atomically flat and continuous, the Ag films display a one-dimensional height modulation along the GaAs[001] direction, leading to a sequence of parallel narrow stripes with a small corrugation of about $0.5 \AA$. These stripes form a quasiperiodic distribution, constituted by a sequence of two characteristic separations of $17 \pm$ $2 \AA$ ("L") and $12 \pm 2 \AA$ ("S") [9,11]. The resulting onedimensional superstructure can be described by a goldenmean Fibonacci sequence of L (long) and S (short) segments, with a coherence length of about $125 \AA$ [11]. Ag on GaAs(110) is the only known system where a singleelement film with such ordering forms on a translationally periodic substrate surface. In contrast to metal films on quasicrystal substrates, where modulated structures grow with different domain orientations [12], Ag films on GaAs exhibit a single domain orientation over an area of macroscopic dimensions.

The low energy electron diffraction (LEED) pattern of the films displays a slightly distorted hexagonal image, close to the one corresponding to the $\operatorname{Ag}(111)$ truncated lattice, and a dense set of unequally spaced spots along the GaAs[001] direction [Fig. 1(a)]. The parameters defining the geometrical relations between the most intense LEED spots are schematically reported in Fig. 1(b). The intensity

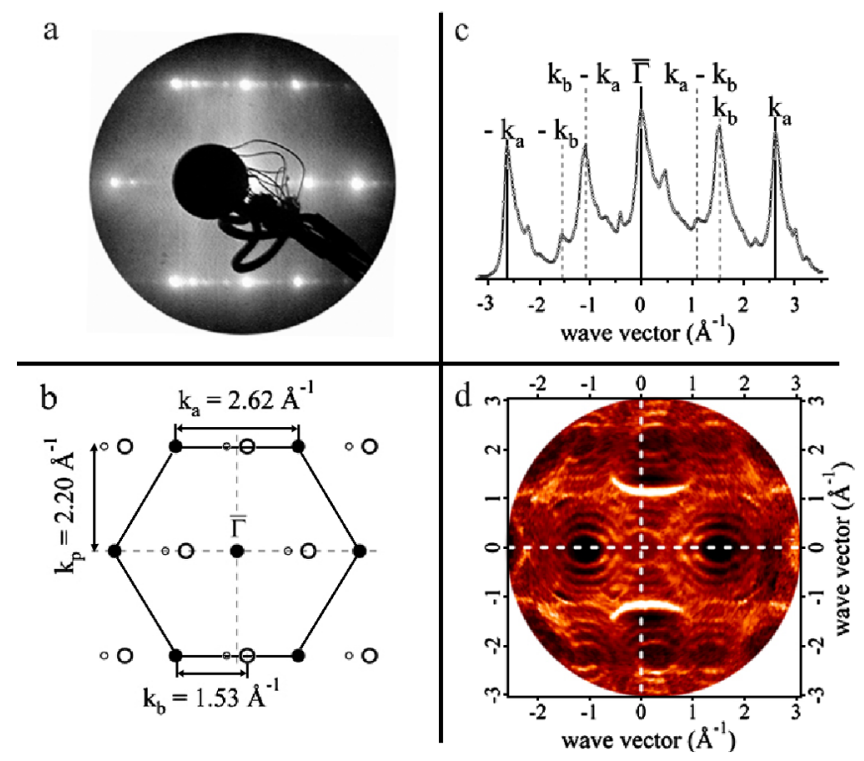

FIG. 1 (color online). (a) LEED pattern of a $19 \AA \mathrm{Ag}$ film on GaAs(110) for $45 \mathrm{eV}$ electron energy. (b) Schematic diagram of the LEED pattern in (a). The circles mark the position of the main spots: filled, unfilled, and unfilled small symbols represent spots of decreasing intensity. (c) LEED intensity along the central row of spots, showing the influence of two periodicities $k_{a}$ and $k_{b}$ on the diffraction pattern. (d) Photoemission intensity pattern at a binding energy of $0.3 \mathrm{eV}$ below $E_{\mathrm{F}}$. The dominant set of concentric ellipses arises from the $s$ - $p$-derived quantum well states of the Ag film. spot profile [Fig. 1(c)] presents a rich fine structure with several well-pronounced features. As indicated in Fig. 1(c), the main spots can be indexed by two vectors, $k_{a}$ and $k_{b}$, with $k_{a}=2.62 \AA^{-1}$, equal to the nearest-neighbor distance of the $\operatorname{Ag}(111)$ reciprocal lattice points stretched by $4 \%$. The second vector is $k_{b}=0.584 k_{a}=1.53 \AA^{-1}$ with the $k_{a} / k_{b}$ ratio close to the golden-mean value $\tau=$ $1.618 \ldots[9,11]$. All diffraction peaks in Fig. 1(c) can be expressed as a linear combination $\left(n k_{a}+m k_{b}\right.$, with $n$ and $m$ integers) of these two vectors within $\pm 0.02 \AA^{-1}$ accuracy. Figure 1(d) shows a photoemission intensity map in two-dimensional reciprocal space, obtained for $s-p \mathrm{Ag}$ states at a binding energy of $0.3 \mathrm{eV}$. From a comparison with the schematic LEED pattern in Fig. 1(c) it is evident that the loci of the centers of the elliptical rings in $k$ space are identical to the major spots in the LEED pattern. The presence of several concentric rings is due to the fact that, because of electron confinement in the direction normal to the surface [13], a discrete number of quantum well (QW) states are formed [14], as discussed in detail below.

Angle-resolved photoemission data of the $s$-p-derived $\mathrm{Ag}$ valence states, measured by varying the polar angle along and perpendicular to the stripes for a film of $26 \AA$ thickness, are shown in Fig. 2. Electron confinement within the $\mathrm{Ag}$ film gives rise to sharp features in the region from the Fermi level $E_{\mathrm{F}}$ up to $3.5 \mathrm{eV}$ binding energy. The occurrence of QW states in this system permits us to study the influence of the intriguing quasiperiodic order on the Ag band structure in great detail, throughout the $s-p$ band region from $E_{\mathrm{F}}$ to the onset of the $d$ bands at $-4 \mathrm{eV}$. In contrast to the surface character of the Shockley-type state giving rise to the prominent feature close to $E_{\mathrm{F}}$ at the $k_{\|}=$ 0 point [15], the QW state charge density is delocalized over the entire film thickness. The QW and surface states at the $k_{\|}=0$ point correspond to the levels observed on this same system in a previous scanning tunneling spectroscopy study [14]. The distinct sequence of energy levels in our photoemission data demonstrates the uniformity of the Ag film thickness on a macroscopic scale.

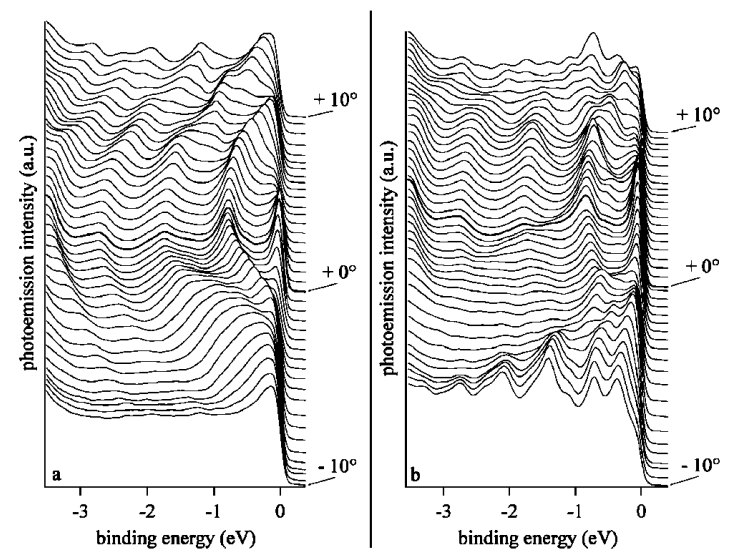

FIG. 2. Sets of photoemission spectra recorded at a photon energy of $45 \mathrm{eV}$ for different polar angles (a) along and (b) across the quasiperiodic stripe structure (see text). 
The $s$ - $p$ states from which the QW states derive have a nearly free electron-like behavior in bulk $\mathrm{Ag}$, and display an almost isotropic two-dimensional dispersion around the $\bar{\Gamma}$ point in crystalline (111) films with an effective mass of $m_{\mathrm{eff}}=0.5 m_{e}$ [16]. In contrast, the dispersion of the QW states in $\mathrm{Ag}$ films on $\mathrm{GaAs}(110)$ exhibits a strongly anisotropic behavior. Near $\theta=0^{\circ}\left(k_{\|}=0\right)$ it closely resembles a free electron-like parabola $\left(m_{\text {eff }}=0.5 m_{e}\right)$ along the direction parallel to the stripes [Fig. 2(a)], but in the quasicrystalline direction it is entirely different, much flatter $\left(m_{\mathrm{eff}}=m_{e}\right)$ and very complex at larger $k_{\|}$values [Fig. 2(b)]. This anisotropy in the effective mass is reflected in the elliptical shape of the constant-energy contours in Fig. 1(d). For a flat two-dimensional $\mathrm{Ag}(111)$ film, the contours would consist of concentric circles. Pure onedimensional electronic states, propagating parallel to the stripe direction and fully localized in the perpendicular direction, would display constant-energy contours consisting of sets of parallel lines. Thus, we find the behavior of QW states in Ag films on GaAs(110) to be between the one- and two-dimensional cases. The stripe geometry of the Ag films on GaAs(110) renders them, indeed, comparable to a lattice of coupled quantum wires or quasi-onedimensional chain systems [17].

The detailed wave vector dependence of the $s$ - $p$ states in the two directions is shown in Fig. 3, in a color rendition of the photoemission intensity for a $19 \AA \mathrm{Ag}$ film over a wider range of $k_{\|}$values. Along the direction parallel to the stripes [Fig. 3(a)], the data define three main groups of parabolas, each comprising a set of QW states. These bands show a direct correspondence with the lattice periodicity along this direction. Two replicas on both sides of $k_{\|}=0$ are located at -2.20 and $+2.20 \AA^{-1}$. They originate at $\vec{k}_{\|}=\left(k_{b}-k_{a} / 2, \pm k_{p}\right)$, i.e., precisely at the location of the intense spots marked on the LEED diagram in Fig. 1(b) [18]. The interaction of neighboring groups of parabolas defines usual Brillouin zone boundaries at $k_{\|}=$ $\pm 1.10 \AA^{-1}$ and produces gap openings between bands of different index " $n$," detected as symmetric intensity modulations at both sides of the zone boundaries.

The QW states in the direction perpendicular to the stripe structure [Fig. 3(b)] are strongly influenced by the quasiperiodic ordering in the films. The set of parabolas repeats at loci centered at -1.09 and $+1.53 \AA^{-1}$, i.e., at unequal and incommensurate intervals. This observation is obviously in sharp contrast to the common electronic behavior of crystalline solids, where the band structure of the first Brillouin zone periodically reproduces itself in each primitive unit cell according to the repeated zone scheme. Here, we observe the QW states to be centered at $k_{\|}$vectors corresponding exactly to two of the strongest peaks in the diffraction pattern, namely, those at the incommensurate wave vectors $\left(k_{b}-k_{a}\right)$ and $k_{b}$. In principle, replicas could be centered at any linear combination $n k_{a}+$ $m k_{b}$. The fact that the spectra are dominated by only a few sets of QW peaks then suggests that only those states
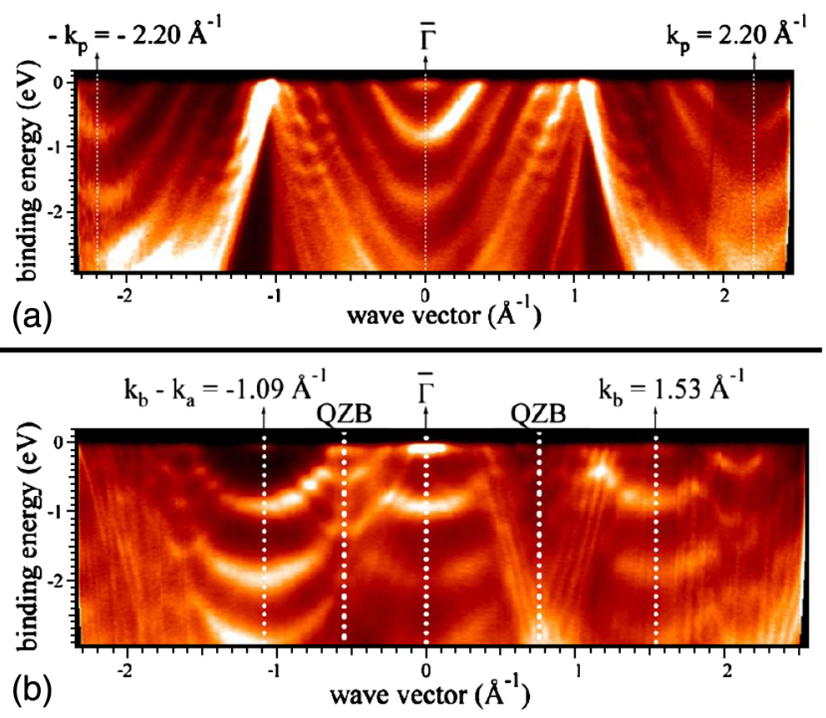

FIG. 3 (color online). Photoemission intensity plot for a wide range of reciprocal lattice vectors, (a) along and (b) across the quasiperiodic stripe structure. The reciprocal lattice vectors corresponding to $k_{p}, k_{b}$, and $k_{b}-k_{a}$ are indicated, together with the location of "quasizone boundaries" (QZBs).

associated with the strongest components of the Fourier expansion of the quasicrystal lattice potential significantly contribute to the spectra. Higher index combinations of the two reciprocal lattice vectors have significantly lower spectral weight in the experimental range, although they may account for several weaker bands detectable only at specific wave vectors. Figure 1 clearly demonstrates the correspondence between the origins of the quantum well paraboloids and the principal wave vectors of the quasicrystal structure.

In periodic systems the strength of the potential manifests itself in the magnitude of the gaps in the band structure at Brillouin zone boundaries. Similarly, in quasicrystals the strongest corresponding gaps are expected at "quasi-Brillouin zone boundaries" located halfway between the main reciprocal lattice points. Figure 4 shows photoemission spectra around $k_{\|}=-0.55 \AA^{-1}$, halfway between $k_{\|}=0$ and $k_{\|}=k_{b}-k_{a}$. The QW states at $\left(k_{b}-\right.$ $\left.k_{a}\right) / 2$ form almost dispersionless bands, separated by gaps on the order of about $400 \mathrm{meV}$. We observe gaps of similar magnitude at all major quasi-Brillouin zone boundaries. Gap openings are intrinsically expected features due to hybridization, as demonstrated for one-dimensional models $[7,19,20]$, but have not until now been observed in three-dimensional quasicrystals. The large energy gaps in the Ag film demonstrate a strongly enhanced influence of the one-dimensional quasicrystalline modulation on the electronic structure compared to the case in the threedimensional bulk quasicrystals.

Since the atomic structure of the Ag film on GaAs(110) is not known in detail, we resort to general arguments to qualitatively explain possible origins for the main features of interest observed in this system. The method of projec- 


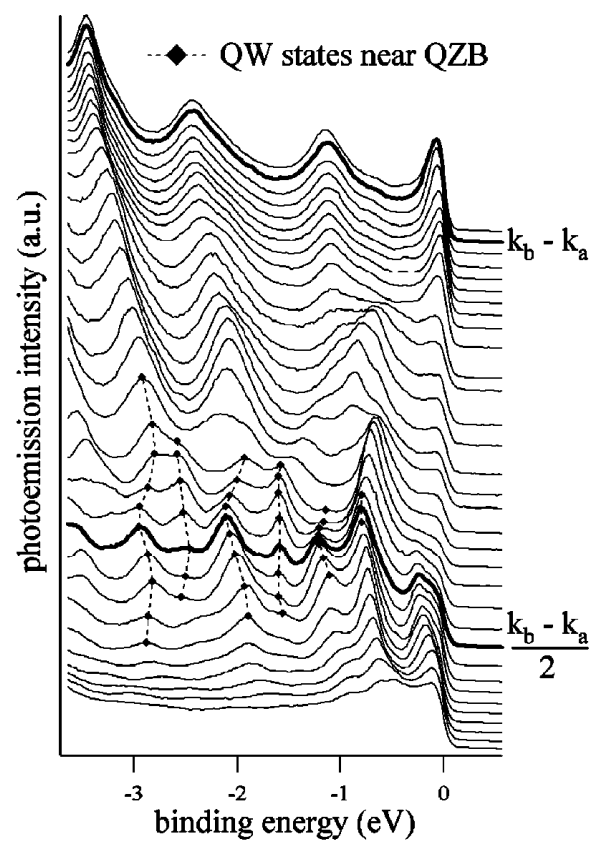

FIG. 4. Close-up spectra of the region around $\left(k_{b}-k_{a}\right) / 2$, demonstrating the occurrence of several gaps between almost nondispersing states at QZB.

tion from higher dimensional spaces [2] can be easily adapted to the present case. If $\mathrm{Ag}$ films (2D) are cut out of bulk crystals (3D) along planes not corresponding to (hkl) netplanes, then quasiperiodic step sequences form at the surfaces and hierarchical LEED patterns with three, instead of the usual two basis vectors [21], are observed. Accordingly, QW parabolas emerge at the surface projection of the center of the bulk Brillouin zones, with hierarchically distributed spectral weights. Gaps open halfway between main (intensity selected) sets of QW parabolas, then defining principal quasi-Brillouin zone boundaries.

With respect to the in-plane electron propagation, a quasiperiodic step structure mainly modifies the atomic coordination at the surface, eventually resulting in effective mass changes for surface related features. However, QW states will be negligibly affected by surface modulations due to their bulk character. The additional structural element, which would be able to account for the observed strongly anisotropic effective mass, is likely to be a Fibonacci sequence of plane defects that propagate from the interface through the entire film thickness. These planes break the phase coherence of electronic states of neighboring blocks of the sequence, giving rise to prominent lateral confinement effects along the quasiperiodic direction.

In conclusion, Ag films on $\mathrm{GaAs}(110)$ exhibit novel and exceptional features related to quasicrystallinity in one dimension. The electronic states display partial onedimensional quantum confinement in the film plane and aperiodically spaced replicas in reciprocal space, in contrast to the repeated zone scheme periodicity of a crystalline structure. Avoided band crossings at quasi-Brillouin zone boundaries demonstrate the strong influence of the one-dimensional quasiperiodic modulation on the electronic structure.

This work is supported as part of the European Science Foundation EUROCORES program SONS under Project MOL-VIC through funds from the Deutsche Forschungsgemeinschaft. K. H. acknowledges support from the Network of Excellence "Complex Metallic Alloys," Contract No. NMP3-CT-2005-500145 from the European Union.

*Present address: Istituto dei Sistemi Complessi, Consiglio Nazionale delle Ricerche, Rome, Italy.

[1] D. Shechtman, I. Blech, D. Gratias, and J. W. Cahn, Phys. Rev. Lett. 53, 1951 (1984).

[2] Physical Properties of Quasicrystals, edited by Z.M. Stadnik (Springer-Verlag, Berlin, 1999).

[3] Eli Rotenberg, W. Theis, K. Horn, and P. Gille, Nature (London) 406, 602 (2000).

[4] W. Theis, Eli Rotenberg, K. J. Franke, P. Gille, and K. Horn, Phys. Rev. B 68, 104205 (2003).

[5] F. Schiller, J. Cordón, D. Vyalikh, A. Rubio, and J.E. Ortega, Phys. Rev. Lett. 94, 016103 (2005).

[6] F. Baumberger, A. Tamai, M. Muntwiler, T. Greber, and J. Osterwalder, Surf. Sci. 532-535, 82 (2003).

[7] J. Voit, L. Perfetti, F. Zwick, H. Berger, G. Margaritondo, G. Grüner, H. Höchst, and M. Grioni, Science 290, 501 (2000).

[8] C. Janot, Quasicrystals: A Primer (Oxford University Press, New York, 1997).

[9] A. R. Smith, K.-J. Chao, Q. Niu, and C.-K. Shih, Science 273, 226 (1996).

[10] G. Neuhold, L. Bartels, J. J. Paggel, and K. Horn, Surf. Sci. 376, 1 (1997); K.-J. Chao, Z. Zhang, Ph. Ebert, and C. K. Shih, Phys. Rev. B 60, 4988 (1999); H. Yu, C. S. Jiang, Ph. Ebert, X.D. Wang, J. M. White, Q. Niu, Z. Zhang, and C. K. Shih, Phys. Rev. Lett. 88, 016102 (2002).

[11] Ph. Ebert, K.-J. Chao, Q. Niu, and C. K. Shih, Phys. Rev. Lett. 83, 3222 (1999).

[12] J. Ledieu, J. T. Hoeft, D. E. Reid, J. A. Smerdon, R. D. Diehl, T. A. Lograsso, A. R. Ross, and R. McGrath, Phys. Rev. Lett. 92, 135507 (2004).

[13] T.-C. Chiang, Surf. Sci. Rep. 39, 181 (2000).

[14] C.-S. Jiang, H.-B. Yu, X.-D. Wang, C.-K. Shih, and Ph. Ebert, Phys. Rev. B 64, 235410 (2001).

[15] G. Nicolay, F. Reinert, S. Hüfner, and P. Blaha, Phys. Rev. B 65, 033407 (2002).

[16] I. Matsuda, T. Ohta, and H. W. Yeom, Phys. Rev. B 65, 085327 (2002).

[17] S. Biermann, A. Georges, A. Lichtenstein, and T. Giamarchi, Phys. Rev. Lett. 87, 276405 (2001).

[18] The periodicity of the system along this direction is equal to $2 k_{p}$, as indicated by the LEED pattern.

[19] M. Grioni, Ch. R. Ast, D. Pacile, M. Papagno, H. Berger, and L. Perfetti, New J. Phys. 7, 106 (2005).

[20] Eli Rotenberg, W. Theis, and K. Horn, J. Alloys Compd. 342, 348 (2002).

[21] The three vectors are the surface projections of the threedimensional basis. 